\title{
UNA APROXIMACIÓN CONCEPTUAL AL TERMINO PASIVOS AMBIENTALES: UNA PROPUESTA PARA SU PUESTA EN PRÁCTICA
}

Adolfo E. Carbal Herrera Universidad de Cartagena Colombia

Carmen Rosales García Universidad de Cartagena Colombia

Alexandra Hernández Viloria Universidad de Cartagena Colombia

Luis Martínez Gómez

Universidad de Cartagena Colombia 
Panorama Económico, Vol. 27 - No. 2 (Abril - Junio de 2019), pp. 497-509

\author{
Adolfo E. Carbal Herrera \\ Carmen Rosales García \\ Alexandra Hernández Viloria \\ Luis Martínez Gómez \\ JEL: Q52, Q56, Q59
}

\title{
Una aproximación conceptual al termino pasivos ambientales: una propuesta para su puesta en práctica
}

\section{Resumen}

En el presente trabajo se aborda un análisis alrededor del término pasivos ambientales, identificando una variedad de conceptos en torno al mismo y de diverso origen. El objeto de la investigación se centra en el planteamiento de un nuevo concepto a partir del análisis y la síntesis de los ya existentes, como base conceptual para el desarrollo de metodologías de medición y valoración en este campo. Metodológicamente se parte de una revisión sistemática de literatura haciendo uso de diversos criterios para la selección del material bibliográfico relevante, la cual permitió identificar las principales nociones sobre el tema, posteriormente, a partir de un ejercicio de análisis y síntesis, en paralelo con la noción contable de pasivo, se propone un nuevo significado. Los resultados muestran una nueva concepción, la cual facilita la puesta en práctica del vocablo, y se avanza teóricamente en la identificación de la responsabilidad y la valoración económica de dichas realidades.

Palabras clave: Pasivos ambientales, Crisis ambiental, Normas Internacionales de Información Financiera, Valoración, medición y reconocimiento.

\section{Une approche conceptuelle du terme passif environnemental: une proposition pour sa mise en œuvre}

\section{Résumé}

Cet article traite d'une analyse autour du terme passif environnemental, identifiant une variété de concepts autour de lui et d'origine diverse. L'objet de la recherche est axé sur l'approche d'un nouveau concept à partir de l'analyse et de la synthèse des concepts existants, comme base conceptuelle pour le développement de méthodologies de mesure et d'évaluation dans ce domaine. D'un point de vue méthodologique, nous partons d'une revue systématique de la littérature utilisant divers critères pour la sélection de matériel bibliographique pertinent, qui nous permet d'identifier les principales notions sur le sujet, ensuite, à partir d'un exercice d'analyse et de synthèse, parallèlement à la notion de comptable de responsabilité, un un nouveau sens est proposé. Les résultats montrent une nouvelle conception, qui facilite la mise en œuvre du mot, et avance théoriquement dans l'identification de la responsabilité et la valorisation économique de ces réalités.

Mots clés: Passifs environnementaux, crise environnementale, normes internationales d'information financière, évaluation, mesure et comptabilisation.

\section{A conceptual approach to the term environmental liabilities: a proposal for its implementation}

\begin{abstract}
This paper deals with an analysis around the term environmental liabilities, identifying a variety of concepts around it and of diverse origin. The object of the research is focused on the approach of a new concept from the analysis and synthesis of the existing ones, as a conceptual base for the development of measurement and assessment methodologies in this field. Methodologically, we start from a systematic literature review using various criteria for the selection of relevant bibliographic material, which allows us to identify the main notions on the subject, subsequently, from an analysis and synthesis exercise, in parallel with the notion liability accountant, a new meaning is proposed.The results show a new conception, which facilitates the implementation of the word, and theoretically advances in the identification of responsibility and the economic valuation of these realities.
\end{abstract}

Keywords: Environmental liabilities, Environmental crisis, International Financial Reporting Standards, Valuation, measurement and recognition. 


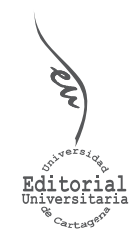

Artículo de Investigación

\title{
Una aproximación conceptual al termino pasivos ambientales: una propuesta para su puesta en práctica
}

\author{
Adolfo E. Carbal Herrera* \\ Universidad de Cartagena, Colombia \\ Carmen Rosales García \\ Universidad de Cartagena, Colombia
}

INFORMACIÓN DEL ARTíCULO

Recepción del artículo: 12/12/2018

Concepto de evaluación: 21/01/2019

Aceptación del artículo: 09/02/2019

\author{
Alexandra Hernández Viloria \\ Universidad de Cartagena, Colombia \\ Luis Martínez Gómez \\ Universidad de Cartagena, Colombia
}

\section{INTRODUCCIÓN}

En el siglo XVIII la sociedad sufrió una serie de transformaciones que orientaron al desarrollo humano en función del capital y el trabajo. La nueva forma de economía trajo consigo la expansión del comercio gracias a las mejoras en la producción, consecuencia de la mecanización, el desarrollo del transporte, entre otros factores. Pero tal progreso ligado a la industrialización también generó efectos menos favorables para la sociedad (Chaves, 2004). La creencia en el crecimiento económico de las naciones como factor para la reducción de la pobreza y solución a las problemáticas sociales fue una falsa promesa; por el contrario, este ha generado una mayor desigualdad. Esta lógica mecanicista e individualista esencia del nuevo modelo de producción, trajo consigo la bifurcación entre la moral y el análisis económico (Weber, 2001; Marcuse, 1969; Naredo, 2003), generando una visión vilipendiada de la naturaleza y promoviendo entonces su excesivo consumo (La Rotta \& Torres, 2017; Castillo, 2011).

Tal cosmovisión constituida en la modernidad desencadenó una crisis ecológica, que con el pasar del tiempo llevó a que las problemáticas sociales y ambientales adquirieran gran magnitud e importancia para la sociedad, al comprender esta, que la mayor causa del deterioro progresivo del ambiente global es el insostenible modelo de producción, el cual conlleva al consumo y explotación desmesurada de los recursos (Colin, 2003), característica de un sistema con una orientación exclusivamente economicista, cuyo eje central es la maximización del beneficio individual en el corto plazo (Segrelles, 2012; Schmidheiny, 1997).

*Autor de correspondencia: acarbalh@unicartagena.edu.co 
Bajo este escenario, a partir de 1970 son muchos los esfuerzos que se han orientado hacia la conciliación del crecimiento económico con la conservación del entorno, dando origen a términos y prácticas como: la gestión ambiental empresarial, la responsabilidad social empresarial entre otros. Estos desarrollos conceptuales y metodológicos pueden entenderse como herramientas que buscan equilibrar la demanda de recursos naturales por parte de la especie humana con la capacidad de producción de la naturaleza (Colby, 1991; González, 2008; Gómez, 2009).

Uno de los vocablos que ha surgido en las últimas décadas, relacionado con la crisis ambiental, es el termino pasivos ambientales, el cual ha sido acuñado para describir "los daños no compensados producidos por una empresa al medio ambiente a lo largo de su historia, en su actividad normal o en caso de accidente" (Russi \& MartinezAlier, 2003, pág. 108), es decir, que estos "representan las obligaciones que tienen las empresas con el ambiente, producto de la contaminación que se desprende de sus procesos de producción" (Rosales \& Quintero, 2015, pág. 123).

Con base en la anterior realidad, el presente trabajo busca profundizar en el término pasivos ambientales, analizar su contenido y las problemáticas asociadas a su puesta en práctica o implementación como herramienta para afrontar la crisis. El documento se compone además de esta introducción, de la descripción del instrumental metodológico empleado para el logro del objetivo trazado, los resultados obtenidos, los cuales muestran de forma sistematizada y clasificados los principales conceptos desarrollados en este campo, la discusión alrededor de las nociones identificadas, la propuesta de una nueva concepción y el abordaje de las principales problemáticas asociadas al termino y que dificultan su puesta en operación.

\section{METODOLOGÍA}

El presente trabajo corresponde fundamentalmente a una revisión sistemática de literatura alrededor del término pasivos ambientales. La investigación se compone de dos momentos, una primera etapa de selección de la información y otra relacionada con un ejercicio de análisis y síntesis de la base conceptual identificada.

Para la primera etapa, se seleccionaron dos bases de datos: Scopus y Redalyc, de acuerdo con la calidad de estas y la cantidad de información que albergan. Los criterios de búsqueda establecidos fueron los siguientes: palabras claves - environmental liabilities, measurement, valuation -, las cuales fueron combinadas estableciendo ecuaciones de búsqueda; el periodo de tiempo fue de 10 años, a partir de 2008 en adelante. Esta primera búsqueda arrojó un resultado de 201 artículos relacionados con el tema. Posteriormente se refinó la pesquisa con base en los siguientes criterios: artículos pertenecientes a revistas ubicadas en el primer y segundo cuartil de la base de datos Scopus, artículos con citación, trabajos sin citación, pero publicados en los dos últimos años, artículos divulgados por revistas en el tercer y cuarto cuartil con citación; y en el caso específico de la base de datos Redalyc, se seleccionaron por su correlación con la temática. Esta preselección permitió la obtención de 129 documentos. Para la selección de la bibliografía se procedió a la lectura de los resúmenes de los trabajos preseleccionados, analizando la importancia y directa relación con la temática a discutir. Seleccionando finalmente un total de 33 títulos. 
La segunda etapa: consistió en el análisis de los documentos seleccionados. La totalidad de los trabajos escogidos fueron sometidos a un análisis interpretativo, a partir de la revisión profunda de cada uno de ellos, esto permitió la sistematización de la información. Dicho examen facilitó la identificación de diferentes concepciones con orígenes disimiles, facilitando su clasificación. También se identificaron los vacíos y falencias en las diferentes nociones de pasivos ambientales al contrastarlas con la noción de pasivo contable, por último, se realizó un ejercicio de síntesis para la formulación de una nueva concepción.

\section{RESULTADOS}

La revisión de literatura en torno al termino pasivos ambientales y responsabilidad ambiental, de acuerdo con su traducción del inglés "Environmental Liability", arrojo como resultado la identificación de una base conceptual en crecimiento, La cual evidencia una importante preocupación por este campo en las últimas tres décadas. Se pudo identificar, que el tema es de interés tanto de organismos multilaterales, institutos de investigación, gobiernos y academia. Los pasivos ambientales al parecer tienen su origen en el vocablo anglosajón "Liability", y el vocablo cobra popularidad a partir de la década de los 90, con el surgimiento del concepto a nivel institucional, derivado de las preocupaciones por los impactos ambientales y daños al medio ambiente generados por la actividad industrial. Lo que conlleva a que se busque establecer la responsabilidad de los causantes y con ello la obligación de responder económicamente por los efectos causados.

A partir del año 2000, los pasivos ambientales, se convierten en un tema de interés para la academia, de acuerdo con la evidencia teórica identificada, la cual muestra un aumento en los trabajos en este campo de origen académico; documentos en los que se identifican a las empresas como las generadoras de tales daños al ambiente.

Por otra parte, los gobiernos latinoamericanos han venido desarrollando una serie de legislaciones entorno a los daños generados al medio ambiente por parte del sector minero, los denominados pasivos ambientales mineros PAM, esto acorde con sus políticas económicas, de carácter extractivista, de los últimos años, que han promovido el auge de la explotación minera dentro de su territorio por parte de empresas extranjeras, poniendo en riesgo ecosistemas estratégicos.

A continuación, se presentan los diversos conceptos de pasivos ambientales identificados, agrupados de acuerdo con su origen: institucionales, academia, legislaciones locales. 
Tabla 1.

Concepciones institucionales del término pasivos ambientales

\begin{tabular}{ll}
\hline \multicolumn{1}{c}{ Autor } & \\
\hline $\begin{array}{l}\text { CEPAL (Citado por } \\
\text { 2012, pág. 126 }\end{array}$ & $\begin{array}{l}\text { Hace referencia a los impactos ambientales generados por las operaciones } \\
\text { mineras abandonadas con o sin dueño u operador identificables y en donde } \\
\text { no se hayan realizado un cierre de minas reglamentado y certificado por la } \\
\text { autoridad correspondiente. }\end{array}$ \\
\hline $\begin{array}{l}\text { Asamblea General } \\
\text { (2013, pág. 9) }\end{array}$ & $\begin{array}{l}\text { Pasivo ambiental contingente: Obligación posible, surgida a raíz de sucesos } \\
\text { pasados, cuya existencia quedará confirmada sólo si llega a ocurrir. Existe } \\
\text { el riesgo de existencia de un pasivo ambiental. }\end{array}$ \\
& $\begin{array}{l}\text { Pasivo ambiental configurado: Obligación existente, surgida a raíz de sucesos } \\
\text { presentes o pasados. Existe un pasivo ambiental legalmente reconocido. }\end{array}$ \\
& $\begin{array}{l}\text { Externalidad: Se aplica esta denominación al resultado de un proceso de } \\
\text { degradación de un ecosistema o ambiente cuando no es posible determinar } \\
\text { un responsable legal identificable del deterioro ambiental. }\end{array}$ \\
\hline $\begin{array}{l}\text { Comisión Europea } \\
\text { (2000) }\end{array}$ & $\begin{array}{l}\text { El término se aplica a los casos en que el daño es el resultado de accidentes } \\
\text { industriales o de la contaminación gradual causada por sustancias peligrosas } \\
\text { odesechos que ingresan al medio ambiente proveniente de fuentes identificables. } \\
\text { Así, la responsabilidad ambiental tiene como objetivo hacer que el causante del } \\
\text { daño ambiental (el contaminador) pague por remediar el daño que ha causado. } \\
\text { Por tanto la regulación ambiental establece normas y procedimientos para } \\
\text { preservar el medio ambiente. }\end{array}$ \\
\hline
\end{tabular}

US EPA (Citado por

Ministerio de Medio

Ambiente, 2000,

pág. 4)
Obligación legal de hacer un gasto en el futuro por actividades realizadas en el presente y el pasado sobre la manufactura, uso, lanzamiento, o amenazas de lanzar, sustancias particulares o actividades que afectan el medio ambiente de manera adversa.
UNITED NATIONS

(2001, pág. 22)
El reconocimiento de los pasivos ambientales tiene como objetivo hacer que el agente que causa el daño ambiental (el que contamina) pague por reparar el daño que han causado.

(IASC, 1998, pág. 2) ${ }^{1}$

Obligación posible, surgida a raíz de sucesos pasados, cuya existencia ha de ser confirmada sólo por la ocurrencia, o en su caso por la no ocurrencia, de uno o más eventos inciertos en el futuro, que no están enteramente bajo el control de la empresa

Fuente: Elaboración propia.

1. Definición acuñada en la Norma Internacional de Contabilidad $N^{\circ} 37$ (NIC 37) la cual trata sobre provisiones, activos y pasivos contingentes 
En este primer grupo de conceptos se pueden identificar aspectos comunes, los cuales podrían sintetizarse bajo la siguiente noción: los pasivos ambientales son daños al medio ambiente ocasionados por operaciones industriales o mineras pasadas que constituyen una obligación en el futuro para su remediación, en donde el responsable es difícil de identificar, situación en la que sería el Estado quien deberá asumir el tratamiento de los mismos, dado que estos se constituyen en lo que algunos investigadores han denominado, pasivos ambientales huérfanos.

Tabla 2.

\section{Concepciones académicas del término pasivos ambientales}

\begin{tabular}{cl}
\hline Autor & Concepto \\
\hline $\begin{array}{l}\text { Russi \& Martinez- } \\
\text { Alier (2003, pág. 108) }\end{array}$ & $\begin{array}{l}\text { La suma de los daños no compensados producidos por una empresa al ambiente } \\
\text { a lo largo de su historia, en su actividad normal o en caso de accidente. Son } \\
\text { deudas hacia la comunidad donde opera. }\end{array}$
\end{tabular}

Martinez-Alier

(2012, pág. 29)
El pasivo ambiental hace referencia a la deuda ecológica no pagada por la empresa durante todo el tiempo de operación, sumada al gasto necesario para impedir daños una vez terminada las operaciones en el ecosistema.

Acosta (2016)

Conjunto de costos económicos de los impactos sociales y ambientales producto de la extracción de recursos naturales en ecosistemas por parte de empresas del sector industrial.

Schneider, Michelon, \& Maier (2017)
Los pasivos ambientales son bastante diferentes de los pasivos financieros. En el caso de incumplimiento de los pasivos financieros, los acreedores terminan con los activos de la empresa y la deuda se libera de manera efectiva. Los pasivos ambientales no desaparecen simplemente si la empresa contaminante entra en insolvencia. Por el contrario, a menudo permanecen con el activo asociado y sirven para perjudicar cualquier flujo de efectivo futuro si a fin de cuenta es responsabilidad de la empresa por su operación dentro del ecosistema.

Fuente: Elaboración propia.

En este segundo grupo, En los casos de, Acosta (2016), Schneider, Michelon, \& Maier (2017) y Rosales \& Quintero (2015), las organizaciones empresariales son identificadas como las responsable de los daños ocasionados a los ecosistemas, por ende, son estas las que deben garantizar la remediación de los recursos afectados o la compensación por los bienes naturales utilizados. Un aspecto común en estos conceptos es la idea de que no solo los sistemas bilógicos son los alterados, también se ponen en riesgo por la operación empresarial, las comunidades aledañas que ven afectada su calidad de vida, por lo que estas se convertirían en sujetos de derechos de acuerdo con estos planteamientos. 
Tabla 3.

Concepciones normativas del término pasivos ambientales

\begin{tabular}{ll}
\hline \multicolumn{1}{c}{ Autor } & Concepto \\
\hline $\begin{array}{l}\text { Ley N } \text { N }^{\circ} 2827 \text { (2004, } \\
\text { art. 2) Perú }\end{array}$ & $\begin{array}{l}\text { Son aquellas instalaciones, efluentes, emisiones, restos o depósitos de residuos } \\
\text { producidos por operaciones mineras, en la actualidad abandonada o inactiva y } \\
\text { que constituyen un riesgo permanente y potencial para la salud de la población, } \\
\text { el ecosistema circundante y la propiedad. }\end{array}$ \\
\hline $\begin{array}{l}\text { Ley Nro. 1333 } 2 \\
\text { Bolivia }\end{array}$ & $\begin{array}{l}\text { Conjunto de impactos negativos perjudiciales para la salud y/o el medio } \\
\text { ambiente, ocasionados por determinadas obras y actividades existentes en } \\
\text { un determinado período de tiempo y los problemas ambientales en general } \\
\text { no solucionados por determinadas obras o actividades. }\end{array}$ \\
$\begin{array}{l}\text { Proyecto De Ley No. } \\
\text { C42 (2014, art. 2) } \\
\text { Colombia }\end{array}$ & $\begin{array}{l}\text { Son considerados pasivos ambientales la obligación de asumir el costo } \\
\text { de un deterioro ambiental del suelo, el aire, el agua o la biodiversidad, } \\
\text { que no fue oportuna y/o adecuadamente mitigado, compensado, manejado, } \\
\text { corregido o reparado, la cual deberá ser asumida por el propietario, poseedor } \\
\text { o tenedor del sitio donde se encuentre, independiente de cualquier acción } \\
\text { civil, sancionatoria o administrativa que el Estado pueda iniciar. }\end{array}$
\end{tabular}

Fuente: Elaboración propia.

Por otra parte, este tercer grupo nos muestra que también es posible un acercamiento a los pasivos ambientales desde los proyectos de ley y legislaciones expedidas en diferentes países de Latinoamérica, como en el caso de Colombia. Sin embargo, en este contexto el abordaje se restringe regularmente al sector minero, como es la realidad de países como Perú y Bolivia, implementándose el término pasivos ambientales mineros PAM. En este caso la regulación que gira en torno al tema, generalmente se centra en las directrices relacionadas con el cierre de minas (FUNDACIÓN FORO NACIONAL POR COLOMBIA, 2013; Oblasser \& Chaparro, 2008).

Por otro lado, merece capítulo aparte para su análisis, lo planteado en las Normas Internacionales de Información Financiera con respecto a los pasivos ambientales, dado que estas se han convertido en referente para el tratamiento de lo que en el plano práctico han denominado provisiones ambientales, de acuerdo con lo plasmado en la NIC 37 (Masanet, Llul \& Rovira, 2008). En este sentido Vargas, (2016, pág. 52) expresa:

Un pasivo de acuerdo con la Norma Internacional es la obligación que posee una entidad, la cual espera desprenderse de beneficios futuros. Las empresas que se dedican a la explotación de recursos minerales legalmente se encuentran obligadas a realizar los procesos de desmantelamiento y restauración. Por lo tanto, estas empresas (las mineras) deberían reconocer 
en sus estados financieros dichas obligaciones o por lo menos revelar en los mismos la obligación que poseen con el Estado Colombiano, en el caso de no ser posible estimar los costos correspondientes.

Como se puede observar la presente norma estableces los requerimientos para el reconocimiento de las provisiones que no sean abordadas en otras normas de este tipo. Estos requisitos están relacionados con obligaciones legales o implícitas, la certeza del desprendimiento de recursos en el futuro y la posibilidad de determinar fiablemente la cuantía por la cual se debe responder. La ausencia de normas que regulen este aspecto ha facilitado la adopción de la NIC 37 por parte de las empresas para el reconocimiento y valoración de sus efectos negativos sobre el ambiente. Es importante exponer que esta es un estándar financiero diseñada bajo criterios disimiles al de la naturaleza y la sostenibilidad, en donde su carácter financiero prevalece sobre el equilibrio ecosistémico.

\section{DISCUSIÓN DE LOS RESULTADOS}

Los conceptos revisados anteriormente se muestran incompletos, lo cual dificulta la puesta en práctica del término. Las nociones sobre pasivos ambientales anteriormente revisadas en su gran mayoría no permiten identificar los dos elementos que constituyen la relación dual que subyace en el pasivo contable, tal dualidad se entiende como "la propiedad bidimensional de las transacciones económicas que permite establecer conexiones específicas entre los distintos elementos patrimoniales y en la relación causa-efecto que se produce como explicación de las variaciones entre inversión y financiación" (Ijiri citado por García, 2010, pág. 30).

Se infiere entonces, que desde esta base conceptual se dificulta el tratamiento de los pasivos ambientales en términos de medición, valoración y reconocimiento de los mismo. En palabras de Sartori, citado por Mejía (2010, pág. 107) "sin conceptos no hay medición, hay que disponer de estos y su análisis lógico antes de poder atribuir un numero con la ayuda de la operación empírica de la medición, la formación de conceptos está antes que la cuantificación y la condiciona".

Tengamos presente que los pasivos contables implican una relación lineal y dual entre un agente con derechos de cobro y otro con una obligación de pago, estos son identificables y cuantificables en unidades monetarias, provienen de un hecho económico a partir del cual se obtuvieron recursos para financiar activos o las actividades de una organización, y representan una obligación presente que debe pagarse en el futuro (Córdova, 2016; Rojas, 2013). Si extrapolamos esta noción al campo del medio ambiente, entonces debemos entender por pasivo ambiental: "la responsabilidad u obligación contraída por una organización, que implica un pago a futuro a terceros, derivado de un hecho o acción pasada que ha generado un impacto que representa una afectación al ambiente, constituyéndose en una alteración a los ecosistemas, con efectos negativos en las comunidades adyacentes al área afectada".

De acuerdo con la noción anterior, planteada por los autores, los pasivos ambientales deben poseer las siguientes características: 
- Representan obligaciones o deudas presentes con terceros.

- Derivadas de un hecho material que genera un impacto que constituye un daño al ambiente.

- La obligación debe pagarse en el futuro.

- Se puede identificar el responsable y cuantificar su valor aproximado.

Esta nueva concepción se muestra más completa y facilita la puesta en práctica del término, sin embargo, el abordaje de los pasivos ambientales es complejo, y no solo depende del establecimiento de una base conceptual y el desarrollo de una metodología de medición y valoración; es vital, que el Estado garantice un marco jurídico para el tratamiento de esta realidad y constituya un sistema robusto de control que identifique, evalué y realice seguimiento al estado de los recursos naturales dentro del territorio nacional.

En este sentido el Estado colombiano debe avanzar en los siguientes aspectos con el propósito de facilitar el tratamiento de los pasivos ambientales:

- Consolidar un marco jurídico para la gestión de los pasivos ambientales

- Levantar un inventario de los recursos naturales, y

- Valorar en términos monetarios dichos recursos.

Algunos autores, como Martínez Alier (2002) afirman que existen dos aspectos complejos en el abordaje de los pasivos ambientales, uno la valoración monetaria y el otro la responsabilidad jurídica. El primero implica el reconocimiento de la complejidad del valor de la naturaleza y la imposibilidad de la determinación de este en términos monetarios, pero como la ciencia no es caso de exactitud, si no de aproximación, es importante avanzar en el desarrollo de metodologías que permitan expresar la integralidad de dicho valor, para expresar una mejor aproximación de este, a diferencia de los métodos basados en una orientación de economía de mercado. Como habíamos dicho anteriormente toda medición, requiere previamente de una base conceptual, lo que nos debe llevar a una construcción teórica sobre el valor de la naturaleza, desde un paradigma diferente al financiero, en este caso el paradigma de la sostenibilidad es una excelente alternativa.

En cuanto a la responsabilidad jurídica, depende del Estado, quien debe legislar de forma estricta para que los contaminadores o quienes hagan uso de los bienes y servicios de los ecosistemas respondan financieramente por los daños causados a estos y a las comunidades afectadas por dicho impacto.

En el campo de los pasivos ambientales aún hay mucho por hacer, pero es necesario avanzar, y desde la investigación científica suministrar conocimiento que oriente las decisiones del Estado en materia de regulación. En este sentido es pertinente plantear cuales son los elementos que constituyen el pasivo ambiental, tomando como referente el concepto planteado por los autores. Para calcular el valor económico de un pasivo ambiental se deben tener en cuanta tres aspectos:

- El costo de recuperación del daño causado.

- El valor monetario de los bienes y servicios ambientales perdidos

- Y la compensación a las comunidades afectadas. 
Y su reconocimiento debe descansar bajo un principio de materialidad, entendido como el hecho real que define la existencia de este, el cual se puede identificar empíricamente, haciendo posible su medición y cuantificación.

\section{CONCLUSIONES}

El grupo de conceptos revisados en este trabajo sobre pasivos ambientales, con diferentes fuentes de origen, se muestran incompletos dificultando el abordaje y tratamiento de tales realidades, dado que estas nociones no contemplan o dificultan la identificación de los elementos duales que constituyen la concepción del pasivo contable, afectando su medición. He aquí donde el presente trabajo se reviste de importancia, al aportar un nuevo concepto, más acabado, que facilita su puesta en práctica u operatividad.

Sin embargo, es importante señalar que, para avanzar en el tratamiento de esta realidad, el Estado debe legislar de forma estricta para establecer la responsabilidad jurídica de quienes causan daños al ambiente, quienes deberán responder financieramente. Además, este debe vigilar y controlar para exigir el cumplimiento de dicha obligación y la compensación a las comunidades afectadas. Ósea, que es el marco jurídico establecido por el Estado el que garantizará la gestión de los pasivos ambientales, al permitir la identificación, de acuerdo con la ley, del agente de derechos de cobro y el agente obligado de pago.

También es importante anotar que este es un campo en desarrollo, del cual queda mucha tela por cortar, en el que se debe avanzar en la construcción teórica del concepto del valor de la naturaleza, el desarrollo de metodologías de medición y valoración soportadas en un paradigma diferente al financiero, para poder expresar un valor de los recursos naturales de una forma más aproximada. En este sentido el presente trabajo propone toda un área de investigación científica en torno al desarrollo teórico y metodológico de esta temática e invita a que profesionales de diversas disciplinas se sumen.

\section{REFERENCIAS BIBLIOGRAFICAS}

Acosta, A. (2016). Maldiciones, herejías y otros milagros de la economía extractivista. Tabula Rasa, 25-55.

Arango, M., \& Olaya, Y. (2012). Problemática de los pasivos ambientales mineros en Colombia.15(3), 125-133.

Asamblea General Ordinaria OLACEFS. (2013). Pasivos Ambientales como efecto de la destrucción de la biodiversidad. Santiago de Chile.

Castillo, P. (2011). Politica economía: crecimiento económico, desarrollo económico, desarrollo sostenible. Revista Internacional del Mundo Económico y del Derecho, 3, 1-2.

Chaves, J. (2004). Desarrollo tecnológico en la primera revolución industrial. Norba. Revista de historia. 17, 93-109. 
Colby, M. (1991). Environmental management in development: the evolution of paradigms. Ecological Economics.

Colin, L. (2003). Deterioro ambiental vs. Desarrollo económico y social. México: Boletin IIE del Instituto de Investigaciones Eléctricas.

Comisión Europea. (2000). White paper on enviromental liability. Luxembourg.

Córdova, V. (2016). Los costos de producción y la rentabilidad de la empresa Mundo Azul. Universidad Técnica de Ambato.

Escobar-Espinoza, A., Guevara-Castañeda, D., \& Uribe-Veloza, M. (2017). Modelos Computacionales y Análisis de la Política Económica en Colombia. Panorama Económico, 25(4), 535-558. doi:https://doi.org/10.32997/24630470-vol.25-num.4-2017-2088

FUNDACIÓN FORO NACIONAL POR COLOMBIA. (2013). La normativa minera en países de América Latina. Un estudio sobre Bolivia, Brasil, Chile, Colombia, Ecuador, México y Perú. Bogotá.

García, I. (2010). Sintesis de la ciencia contable. Universidad de Salamanca.

Gómez, M. (2009). Tensiones, posibilidades y riesgos de la contabilidad medioambiental empresarial. (Una síntesis de su evolución). Universidad de Antioquia.

González, A. (2008). Diseño de metodología para la identificación de pasivos ambientales. Medellín: Universidad Nacional de Colombia.

Ijiri, Y. (1967). The foundations of accounting measurement. New Jersey: Prentice Hall.

La Rotta, Á., \& Torres, M. (2017). Explotación minera y sus impactos ambientales y en salud. El caso de Potosí en Bogotá. SAÚDE DEBATE, 77-91.

Ley $\mathrm{N}^{\circ}$ 28271. (2004). Ley que regula los pasivos ambientales de la actividad minera. Perú: Congreso de Perú.

Ley No. 1333. (1992). Ley Del Medio Ambiente. Bolivia: Congreso de Bolivia.

Marcuse, H. (1969). La sociedad industrial y el marxismo. Buenos Aires, Argentina: Editorial Quintaria .

Martinez-Alier, J. (2012). Cuantificación de la deuda ecológica. Gestión y ambiente, 23-34.

Masanet, M. J., Llul, A., \& Rovira, M. R. (2008). La información medioambiental en el marco de las NIIF y la regulación española. Su aplicación al caso de los pasivos. Revista de Contabilidad y Dirección, 135-162.

Ministerio de Medio Ambiente. (2000). Dimensionamiento de los pasivos ambientales en Colombia una visión nacional y regional hacia el futuro. Memorias talleres regionales año 2000. 
Missaglia, M. (2015). ¿Se pueden aplicar las ideas Keynesianas al largo plazo? Unas reflexiones teóricas y un modelo ilustrativo. Panorama Económico, 23, 1730. DOI: https://doi.org/10.32997/2463-0470-vol.23-num.1-2015-1380

Naredo, J. (2003). La economía en evolución: Historia y perspectivas de las categorias básicas del pensamiento económico. Madrid, España: SIglo XXI de España Editores S.A.

Oblasser, A., \& Chaparro, E. (2008). Estudio comparativo de la gestión de los pasivos ambientales mineros en Bolivia, Chile, Perú y Estados Unidos. División de Recursos Naturales e Infraestructura. CEPAL.

Proyecto de Ley $\mathrm{N}^{\circ}$ 042. (2014). Por medio de la cual se regula los pasivos ambientales, y se dictan otras disposiciones. Colombia: Congreso de Colombia.

Rojas, J. (2013). Aportes y vacíos de la contabilidad en el reconocimiento y valoración de los pasivos ambientales de las empresas. La contabilidad saber estratégico y soporte del desarrollo social y económico. Cartagena, Colombia: Universidad Libre Sede Cartagena.

Rosales, M., \& Quintero, M. (2015). Pasivos ambientales y las Normas Internacionales de Información Financiera en las PYMES manufactureras del municipio Libertador del estado Mérida. Actualidad Contable FACES, 123-141.

Russi, D., \& Martinez, J. (2003). Los pasivos ambientales. ICONOS. Revista especializada en ciencias sociales de la Facultad Latinoamericana de Ciencias Sociales Sede Ecuador, 123-131.

Schmidheiny, S. (1997). Cambiando el rumbo. Una perspectiva global del empresario para el desarrollo y el medio ambiente. Fondo de Cultura Económica.

Schneider, T., Michelon, G., \& Maier, M. (2017). Environmental liabilities and diversity in practice under international financial reporting standards. Accounting, Auditing \& Accountability Journal, 378-403.

Segrelles, J. A. (2012). La ecología y el desarrollo sostenible frente al capitalismo: Una contradicción insuperable. Universidad de Alicante (España) .

United Nations. (2001). Environmental Management Accounting Procedures and Principles. New York.

Vargas, C. (2016). Tratamiento contable bajo NIIF del pasivo ambiental que conlleva la explotación de minería de carbón en Colombia. Bogotá D.C.

Vergara-De la Ossa, R., Londoño-Vega, C., Pérez-Benítez, N., \& Torres-Castellar, R. (2015). La adopción de las Normas Internacionales de Información Financiera en Colombia. Panorama Económico, 23, 119-132. DOI: https://doi.org/10.32997/ 2463-0470-vol.23-num.1-2015-1382

Weber, M. (2001). Historia económica general. Octava reimpresión. México D.F., México: Fondo de Cultura Económica de México . 